\title{
Phlebotomine mortality effect of systemic insecticides administered to dogs
}

Sonia Ares Gomez ${ }^{1 *}$ D, Javier Lucientes Curdi ${ }^{2}$, Juan Antonio Castillo Hernandez ${ }^{2}$, Paz Peris Peris ${ }^{2}$, Adriana Esteban Gil $^{2}$, Ronald Vladimir Oropeza Velasquez ${ }^{2}$, Paula Ortega Hernandez ${ }^{2}$ and Albert Picado ${ }^{1}$

\begin{abstract}
Background: Zoonotic visceral leishmaniasis (ZVL) caused by Leishmania (Leishmania) infantum is an important disease in humans and dogs. Different mammal species are reservoirs but dogs are considered to be the main one. Phlebotomine sand flies are the proven vector. Four systemic insecticides approved for their use in dogs were previously selected based on their potential to be used in endemic countries as part of the control programs of $Z V L$. These insecticides are proved to be safe and effective against the on-label insects and parasites, but there is no information about their activity against phlebotomine sand flies.
\end{abstract}

Methods: The phlebotomine mortality of four systemic insecticides in dogs was evaluated using two randomized clinical trials. For the first trial, thirty dogs were randomly allocated into five groups: four treatments and one control, of equal size. The treatments evaluated were: Guardian ${ }^{\oplus S R}$, Elanco (moxidectin); Comfortis ${ }^{\oplus}$, Elanco (spinosad); Bravecto ${ }^{\oplus}$, Merck Animal Health (fluralaner); and NexGard ${ }^{\oplus}$, Merial (afoxolaner). Blood from dogs was taken at days 2, 4, 21 and 31 post-treatment (trial 1). The compound that showed the highest efficacy was selected for a second trial (trial 2) with 20 dogs sampled at days $0,2,4,7,14,18,32,39,51$ and 84 post-treatment. Membrane feeding bioassays with Phlebotomus papatasi were used to evaluate the phlebotomine mortality efficacy of the different treatments. Phlebotomine mortality was observed every $24 \mathrm{~h}$ following the membrane feeding during 5 days. A mixed model for a negative binomial logistic regression, and a Cox proportional hazard mixed model were used to estimate phlebotomine mortality due to different treatments.

Results: Fluralaner was the only compound that showed significant phlebotomine mortality. Fluralaner maintained the phlebotomine mortality between $60-80 \%$ for 30 days after treatment. In trial 1 we found that fluralaner increased the risk of death by 1.9 times ( $95 \% \mathrm{Cl}: 1.02-3.6)$ and 1.7 times (95\% Cl: 1.09-2.6) at days 2 and 4 after treatment. The Cox model resulted in an increase of 1.47 (95\% Cl: 1.1-1.96) times in hazard risk at day 2 and 1.89 (95\% Cl: 1.35-2.45) at day 4 after treatment.

In trial 2 we found that fluralaner increased the risk of death by 1.64 times (95\% Cl: 1.16-2.54) and 1.97 times (95\% Cl: 1. 23-3.17) at days 14 and 32. The hazard risk was also increased by 1.92 (95\% Cl: 1.4-2.64) times at day 14 after treatment. Phlebotomine survival including all experimental days was significantly lower in the fluralaner group in both trials.

Conclusions: A single oral treatment of fluralaner in dogs induces phlebotomine mortality. Systemic insecticides in dogs should be considered as a potential preventive measure of ZVL.

Keywords: Zoonotic visceral leishmaniasis (ZVL), Sand flies, Dogs, Systemic insecticides

\footnotetext{
*Correspondence: soniaares@gmail.com; sonia.ares@isglobal.org

'ISGlobal, Barcelona Ctr. Int. Health Res. (CRESIB), Hospital Clínic-Universitat

de Barcelona, Barcelona, Spain

Full list of author information is available at the end of the article
} 


\section{Background}

Zoonotic visceral leishmaniasis (ZVL) is a public health concern in some regions (e.g. Brazil, Mediterranean basin, Iran and China) where it causes a significant number of human cases, particularly in children [1]. The disease can be lethal if appropriate treatment is not provided [2]. The causal agent is a protozoan parasite named Leishmania (Leishmania) infantum. Different mammal species are reservoirs but dogs are considered to be the main one [3] and phlebotomine sand flies are the proven vector [4]. Current control measures (e.g. culling of infected dogs, insecticide spraying, dog collars) have a limited impact on ZVL transmission for several reasons [5]. New costeffective vector control methods are required. Systemic insecticides that are easily administered to dogs (e.g. orally) and kill sand flies biting on them for a significant amount of time, could be used as an alternative or complement to control ZVL in endemic regions [6].

A number of systemic insecticides for dogs are currently available. These are mainly registered to treat and prevent ectoparasitic infestations such as fleas, ticks, and mites [7-9]. Others are used to prevent dirofilarial infections in dogs [10]. Systemic insecticides enter into the circulatory system to be distributed through the entire body. The route of administration can be oral, parenteral, or topical [11].

The compounds considered as systemic insecticides currently used in dogs are isoxazolines (fluralaner, afoxoloner, sarolaner), spinosyns (spinosad), neonicotinoids (nitenpyram), and macrocyclic lactones (ivermectin, selamectin, moxidectin, and doramectin). Isoxazolines are antagonist of the gamma-aminobutyric acid (GABA)gated chloride channels. Several studies have demonstrated the isoxazolines efficacy against fleas and ticks infestations after a single oral administration [12-14]. The duration of the effect ranges from four (afoxoloner, sarolaner) to 12 weeks (fluralaner). Macrocyclic lactones act as GABA agonists and agonists of the glutamategated chloride channels. Systemically distributed macrocylic lactones are used to control heartworm infections in dogs (Dirofilaria immitis) [15].

Some of these insecticides have phlebotomine mortality effect as shown in experimental $[16,17]$ and field studies [18]. There is, however, no evidence of their effect on sand flies when administered to dogs. In this study we evaluate the phlebotomine mortality effect post-feeding in blood of dogs treated with four systemic insecticides: moxidectin, spinosad, fluralaner and afoxolaner.

\section{Methods}

\section{Systemic insecticides}

The insecticides evaluated in this study were selected based on their: (i) phlebotomine mortality showed in laboratory experiments or a potential phlebotomine mortality based on the insecticide mechanism of action; (ii) optimal plasma concentration for the phlebotomine mortality effect based on in vivo or in vitro studies; (iii) long-lasting action (> 12 weeks); (iv) ease of administration (e.g. oral); and (v) availability as commercial products for dogs [6]. The four compounds evaluated were: Guardian ${ }^{\circ}$ SR, Elanco (moxidectin); Comfortis ${ }^{\circ}$, Elanco (spinosad); Bravecto ${ }^{\circ}$, Merck Animal Health (fluralaner); and NexGard ${ }^{\circ}$, Merial (afoxolaner). All the products where administered just once and following the label indications: Guardian ${ }^{\circ} \mathrm{SR}$, subcutaneous injection dose of $0.17 \mathrm{mg} / \mathrm{kg}$ body weight; Comfortis ${ }^{\circ}$, chewable table 425 mg for dogs > 6-9.4 kg; Bravecto ${ }^{\circ}$, chewable tablet 500 $\mathrm{mg}$ for dogs $>4.5-10 \mathrm{~kg}$, and NexGard ${ }^{\oplus}$, chewable tablet $28.3 \mathrm{mg}$ for dogs $>4-10 \mathrm{~kg}$. Fluralaner was administered on fasted dogs in trial 1 and on fed dogs in trial 2 as it has shown to increase bioavailability [19].

\section{Study design}

The study was run between February 2016 and June 2017. Two randomized controlled trials were conducted in dogs. The first clinical trial (trial 1) was designed to evaluate the effect of four systemic insecticides (see above). The compound showing the best performance in trial 1 was further evaluated in the second clinical trial (trial 2). Trial 2 was designed to measure the phlebotomine mortality of the selected compounds for three months. The phlebotomine mortality of the systemic insecticides was measured by comparing the mortality of sand flies feeding on blood from treated and control dogs.

\section{Study site and dogs}

The study was conducted in one breeding kennel located in the Province of Huesca (Spain). Dogs from 1-5 yearsold, from the breeds Parson Rusell (trial 1: $n=7$; trial 2: $n=11$ ), Jack Rusell (trial 1: $n=13$; trial 2: $n=9$ ) and Teckel (trail 1: $n=10$ ) participated in the study. To avoid contamination, no insecticide treatment was provided to the dogs or the kennel in the three months preceding the study. All of the dogs each had a microchip and were examined by a veterinarian at the start of the study. Only healthy dogs were enrolled. The enrolled dogs were kept in the kennel with their regular routines and diet for the whole study period.

\section{Sample size}

The sample size calculation in trial 1 aimed at detecting at least a $65 \%$ increase in phlebotomine mortality at 24 $\mathrm{h}$ after feeding on a treated dog's blood collected up to 30 days post-treatment. Trial 2 was powered to detect at least $45 \%$ increase in phlebotomine mortality $24 \mathrm{~h}$ after feeding on a treated dog's blood up to 90 days posttreatment. These calculations were done assuming $20 \%$ 
phlebotomine mortality in control group, alpha error of 0.05 and $80 \%$ power [20]. The sample size calculation resulted in 6 dogs per treatment group in trial 1 (24 dogs in 4 treatment groups of equal size and 6 dogs in control) and 10 dogs per treatment group in trial 2 (10 dogs in treatment and 10 dogs in control).

\section{Randomization and allocation}

We selected dogs (trial 1: $n=30$; trial 2: $n=20$ ) of similar size from the kennel that participated in the study. Once the dogs were selected the randomization was done using the dogs' microchip numbers. To achieve balance across treatment groups a stratified permuted block randomization was used [21]. Dogs were subdivide into strata (breed and gender) then a permuted block randomization was used for each stratum [22]. The permuted block randomization was performed using the sample function in $\mathrm{R}$ (version 3 . 3) [23]. The only person aware of the randomization results was the researcher who administered the treatments. Researchers responsible for taking the blood samples and performing the membrane feedings were blind to the treatment allocation.

Treatments in the intervention groups were administered in a unique dose at Day 0 following the label indications. Comfortis ${ }^{\oplus}$, Bravecto ${ }^{\circ}$, and $\mathrm{NexGard}^{\circ}$ were given orally and Guardian ${ }^{\ominus}$ SR was administered subcutaneously. The control dogs received no treatment.

Blood samples from the cephalic or jugular veins were taken on days 2, 4, 21 and 31 post-treatment in trial 1 and on days $0,2,4,7,14,18,32,39,51$ and 84 in trial 2 (Additional file 1: Figure S1). At each time-point $4 \mathrm{ml}$ of blood per dog was kept in heparinized tubes preserved at $-21{ }^{\circ} \mathrm{C}$ for $3-9$ months until the bioassays were performed. Blood samples were identified with the date of collection and the dog's microchip number.

\section{Outcomes}

The effect of the four systemic insecticides was measured by membrane feeding bioassays using reared Phlebotomus papatasi sand flies from the School of Veterinary Medicine at the University of Zaragoza. To summarize, the colony was maintained at $30{ }^{\circ} \mathrm{C}, 90 \%$ relative humidity, and a photo period of $17: 7 \mathrm{~h}$ (light: dark photocycle). The sand flies were deprived of sucrose solution $12 \mathrm{~h}$ before the bioassays. The dog's blood was thawed at room temperature $\left(23{ }^{\circ} \mathrm{C}\right)$ on the day of the bioassay. Two- to seven-days-old $P$. papatasi sand flies were exposed to the blood collected from the dogs in the study using Hemotek (Discovery Workshops, Lancashire, UK [24]). Hemotek kept the blood at $37{ }^{\circ} \mathrm{C}$ and chicken skin membrane was used to mimic bloodfeeding on-animal conditions.
The membrane feeding bioassays for each blood sample were performed with around 40 females of $P$. papatasi mixed with some males. The sand flies were allowed to feed on the dog's blood for at least $1.5 \mathrm{~h}$ or until 15 females were observed to be fully engorged; a total of 1800 fully engorged sand flies were needed for trial 1 and 3000 for trial 2.

After the membrane feeding, the 15 fully engorged sand flies were placed into individual plastic cups of 90 $\mathrm{ml}$ and $60 \mathrm{~mm}$ diameter. An orifice of $45 \mathrm{~mm}$ was made in the bottom of the cup and covered with clay to keep humidity high inside the cup. The selection of the fully engorged sand flies was made by visual observation. Sucrose solution was provided to all sand flies. Phlebotomine mortality was recorded every $24 \mathrm{~h}$ for five days.

\section{Statistical analysis}

The average mortality and its 95\% confidence interval (CI) of the 15 fully engorged sand flies were calculated per treatment group (control, afoxolaner, flularaner, moxidectin and spinosad), sampling day (trial 1: 2, 4, 21 and 31; and trial 2: days $0,2,4,7,14,18,32,39,51$ and 84) and mortality recording time (24, 48, 72, 96 and $120 \mathrm{~h})$.

Two different statistical analyses were used to assess the anti-phlebotomine mortality of systemic insecticides. First, a mixed model for a negative binomial logistic regression [25] was performed to estimate differences in phlebotomine mortality $24 \mathrm{~h}$ after blood ingestion between treatment and control groups over time (30 days in trial 1 and 60 days in trial 2). The model analyzed the incidence rate (IR) as the response variable, that is, the number of fully engorged sand flies dead in $24 \mathrm{~h}$ given the total number of fully engorged sand flies (offset). The exponential of the model estimates are the incidence rate ratios (IRR) comparing each treatment to control. The variable dog was included as a random effect to account for the repeated sampling. Sampling day, treatment, and their interaction were the explanatory variables. The interaction term was included to be able to capture differences among the treatments in their efficacy over time (e.g. one treatment could have the highest effect 2 days after administration but another treatment could have the highest effect 4 days after administration). Secondly, survival analyses were conducted. Kaplan Meier curves were used to represent the phlebotomine mortality up to 5 days post-feeding for treatment and control groups. Then, a Cox proportional hazard mixed model for the number of dead sand flies was used to estimate the mean increase in the risk of phlebotomine mortality per day in treated groups compared to control. The Cox proportional hazard model response variable is the effect of treatment in the hazard rate of a phlebotomine dying (i.e. probability of death in $24 \mathrm{~h}$ ) after feeding on a dog's blood. The exponential of 
the model estimates are the hazard rate ratios (HRR) comparing each treatment to control. As for the negative binomial model, dog was included as a random effect and sampling day, treatment, and their interaction were the explanatory variables. The z-statistic was used to determine statistical significance of the estimates obtained from both models. Bayesian information criterion (BIC) was used to select the best models.

All the analyses were conducted in $\mathrm{R}$ (version 3.3) [23]. The MASS package [26] was used to conduct de negative binomial regression. The survival and coxme packages $[27,28]$ were used to conduct the Kaplan Meier curves and the Cox proportional hazard mixed model respectively.

\section{Results}

\section{Trial 1}

Six dogs per group were included in trial 1 . Gender and breed were evenly distributed among groups (Additional file 2: Table S1). No adverse effects related to the administration of the treatments were observed in any dog.

The total number of fully engorged sand flies evaluated was 1700 . The total number of sand flies used per treatment groups was 360, 316, 365, 330 and 329 for control, afoxolaner, fluralaner, moxidectin and spinosad, respectively. Phlebotomine mortality, the corresponding percentage and its $95 \% \mathrm{CI}$ from the mortality observed at $24,48,72,96$ and $120 \mathrm{~h}$ after blood-feeding for treatment and control groups and sampling day are reported in Additional file 2: Table S2.

Compared to control, phlebotomine mortality $24 \mathrm{~h}$ post-feeding was higher in the sand flie group fed in blood samples of dogs treated with fluralaner at days 2 (55\%; 95\% CI: 45-65\%) and 4 (77\%; 95\% CI: 68-86\%) post-insecticide-treatment. The phlebotomine mortality for the fluralaner group was similar to the control group at days 21 (37\%; 95\% CI: 27-47\%) and 31 (43\%; 95\% CI: $32-53 \%)$ post-treatment. No differences were observed in the phlebotomine mortality in the other treatments. The negative binomial mixed model confirmed the previous observations. Treating dogs with fluralaner increased the risk of death for sand flies by 1.9 (95\% CI: 1.02-3.6) and 1.7 (95\% CI: 1.09-2.6) times at day 2 and 4 , respectively, compared to untreated dogs. None of the treatments had an effect on phlebotomine mortality at days 21 and 31 (Table 1 and Fig. 1a).

Kaplan Meier (KM) curves also indicated that fluranaler was the only compound that had a phlebotomine mortality effect. At days 2 and 4 the KM curves showed the biggest difference in phlebotomine survival comparing fluralaner with control at 24 and $48 \mathrm{~h}$ after bloodfeeding. At $80 \mathrm{~h}$ after blood-feeding, phlebotomine survival is between 0 and $20 \%$ with no differences among days or treatment groups (Additional file 3: Figure S2).
Table 1 Trial 1 results from the negative binomial mixed model that included treatment group, sample day and their interaction as fixed effects and dog as random effect. Results are presented as the incidence rate ratio (IRR) which is the increase in the rate of incidence compared with control at each sampling day

\begin{tabular}{llll}
\hline Covariates effect $^{\mathrm{a}}$ & $\mathrm{IRR}^{\mathrm{b}}$ & $95 \% \mathrm{Cl}$ & $P$-value \\
\hline Day 2: Control & Reference & - & - \\
Afoxolaner & 1.39 & $0.74-2.67$ & 0.31 \\
Fluralaner & 1.94 & $1.14-3.39$ & $0.01^{*}$ \\
Moxidectin & 0.60 & $0.26-1.29$ & 0.21 \\
Spinosad & 0.90 & $0.43-1.80$ & 0.77 \\
Day 4: Control & Reference & - & - \\
Afoxolaner & 1.34 & $0.82-2.18$ & 0.23 \\
Fluralaner & 1.68 & $1.05-2.71$ & $0.03^{*}$ \\
Moxidectin & 0.77 & $0.45-1.31$ & 0.34 \\
Spinosad & 1.02 & $0.61-1.70$ & 0.92 \\
Day 21: Control & Reference & - & - \\
Afoxolaner & 1.11 & $0.67-1.89$ & 0.67 \\
Fluralaner & 0.81 & $0.47-1.38$ & 0.45 \\
Moxidectin & 0.91 & $0.54-1.53$ & 0.73 \\
Spinosad & 0.96 & $0.57-1.61$ & 0.89 \\
Day 31: Control & Reference & - & - \\
Afoxolaner & 1.51 & $0.81-2.89$ & 0.20 \\
Fluralaner & 1.21 & $0.64-2.31$ & 0.55 \\
Moxidectin & 1.33 & $0.65-2.74$ & 0.41 \\
Spinosad & 1.41 & $0.77-2.66$ & 0.27 \\
\hline The covalate & & &
\end{tabular}

${ }^{a}$ The covariate effects used control group at each corresponding day after treatment as baseline comparison $[\operatorname{Pr}(>|z|)]$

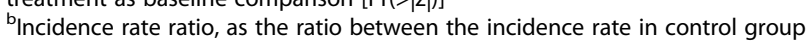
and the incidence rate for each treatment group, at the corresponding sampling day after treatment

*Significance level defined at $a=0.05$

These observations were confirmed by the cox proportional hazard model that showed significant increase in hazard risk (HR) only for the fluralaner group on days 2 and 4 after treatment (Additional file 4: Figure S3). The hazard risk in fluralaner treated dogs was 1.47 (95\% CI: 1.1-1.96) times higher than control at day 2 after treatment. This hazard increased to 1.89 (95\% CI: 1.35-2.45) at day 4 after treatment (Table 2). However, at days 21 and 31 after treatment the effect of fluralaner decreases and the hazard ratio is no longer significantly above 1 (Table 2 and Fig. 2a).

\section{Trial 2}

Ten dogs per group were included in trial 2. Gender and breed were evenly distributed among groups (Additional file 2: Table S1). No adverse effects related to fluranaler administration were observed.

The total number of fully engorged sand flies evaluated was 2982: 1347 for fluralaner and 1635 for the 

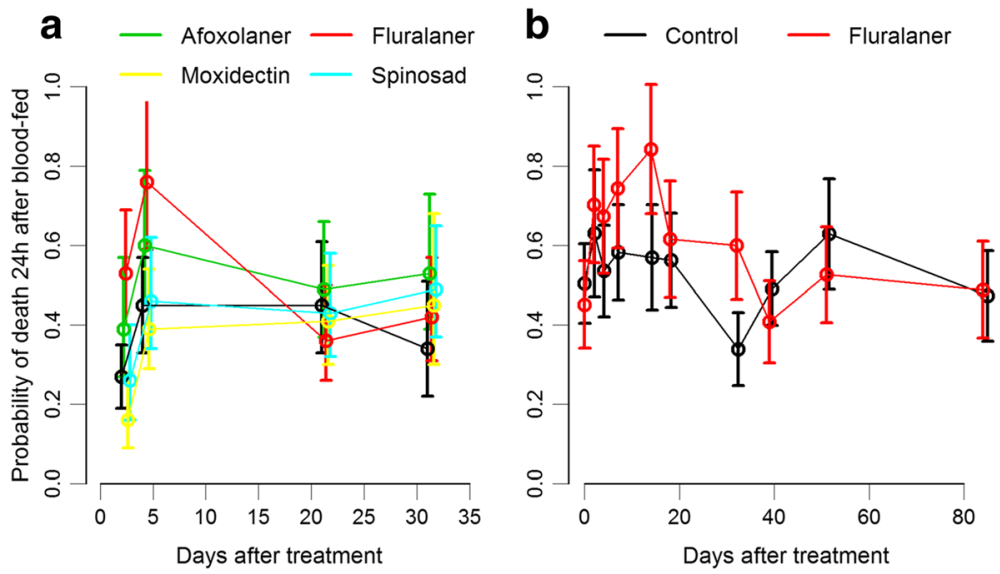

Fig. 1 Probability of death for a phlebotomine $24 \mathrm{~h}$ after blood-feeding for each treatment group and sampling day estimated from a mixed model for a negative binomial logistic regression. The variable dog was included as a random effect, treatement, days after treatment, and their interaction were covariates. a Trial 1: afoxolaner vs fluralaner vs moxidectin vs spinosad vs control. b Trial 2: fluralaner vs control

Table 2 Trial 1 results from the cox proportional hazard model that included treatment group, sample day and their interaction as fixed effects and dog as random effect. Results are presented as the hazard rate ratio (HRR) which is the increase in the hazard rate compared with control at each sampling day

\begin{tabular}{|c|c|c|c|}
\hline Covariates effect $^{a}$ & $\mathrm{HRR}^{\mathrm{b}}$ & $95 \% \mathrm{Cl}$ & $P$-value \\
\hline Day 2: Control & Reference & - & - \\
\hline Afoxolaner & 1.13 & $0.85-1.56$ & 0.45 \\
\hline Fluralaner & 1.47 & $1.10-1.96$ & $<0.001^{*}$ \\
\hline Moxidectin & 0.73 & $0.51-1.01$ & 0.06 \\
\hline Spinosad & 0.76 & $0.54-1.08$ & 0.08 \\
\hline Day 4: Control & Reference & - & - \\
\hline Afoxolaner & 1.25 & $0.93-1.67$ & 0.08 \\
\hline Fluralaner & 1.83 & $1.36-2.45$ & $<0.001^{*}$ \\
\hline Moxidectin & 0.84 & $0.62-1.13$ & 0.17 \\
\hline Spinosad & 0.74 & $0.53-1.02$ & 0.33 \\
\hline Day 21: Control & Reference & - & - \\
\hline Afoxolaner & 1.39 & $0.98-1.86$ & 0.07 \\
\hline Fluralaner & 0.96 & $0.72-1.28$ & 0.91 \\
\hline Moxidectin & 1.06 & $0.78-1.39$ & 0.55 \\
\hline Spinosad & 1.04 & $0.76-1.38$ & 0.61 \\
\hline Day 31: Control & Reference & - & - \\
\hline Afoxolaner & 1.42 & $0.99-1.93$ & 0.08 \\
\hline Fluralaner & 1.25 & $0.93-1.68$ & 0.43 \\
\hline Moxidectin & 1.12 & $0.65-1.64$ & 0.57 \\
\hline Spinosad & 0.99 & $0.73-1.32$ & 0.37 \\
\hline
\end{tabular}

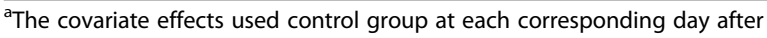
treatment as baseline comparison $[\operatorname{Pr}(>|z|)]$

${ }^{\mathrm{b}}$ Hazard rate ratio, as the ratio between the hazard rate in control group and the hazard rate for each treatment group, at the corresponding sampling day after treatment

"Significance level defined at $\alpha=0.05$ control group. Phlebotomine mortality, the corresponding percentage and its $95 \% \mathrm{CI}$ from the mortality observed at 24, 48, 72, 96 and $120 \mathrm{~h}$ after blood-feeding for treatment and control groups and sampling day are reported in Additional file 2: Table S3.

Compared to the control, phlebotomine mortality $24 \mathrm{~h}$ after blood-feeding was higher for the fluralaner group at days 14 (85\%; 95\% CI: 78-90\%) and 32 (60\%; 95\% CI: 52-68\%) after treatment (Additional file 4: Figure S3). The negative binomial mixed model confirmed that treating dogs with fluralaner increased the risk of death for sand flies by 1.64 (95\% CI: 1.16-2.54) and 1.97 (95\% CI: $1.23-3.17)$ times at day 14 and 32 after treatment respectively (Table 3 and Fig. 1b).

Kaplan Meier (KM) curves also indicated that fluralaner had phlebotomine mortality. For each day of the trial the $\mathrm{KM}$ curve showed the biggest difference in phlebotomine survival at $24 \mathrm{~h}$ after blood-feeding. At $80 \mathrm{~h}$ after bloodfeeding phlebotomine survival is between 0 and $10 \%$ with no differences between fluralaner and control (Additional file 5: Figure S4). The cox proportional hazard model showed significant increase in hazard risk (HR) only on day 14 after treatment. The hazard risk in fluralaner treated dogs was 1.92 (95\% CI: 1.4-2.64) times higher than control at 14 days after treatment (Table 4 and Fig. 2b).

The mixed models of both trials were run with dog as random effect. However, this random effect was significant by likelihood ratio test (chi-square test; trial $1, d f=29 P=$ 4. 5609e-10; trial $2, d f=19, P=6.627005 \mathrm{e}-12$ ) only for the Cox proportional hazard models.

\section{Discussion}

A single dose of Bravecto ${ }^{\circ}$ chewable tablet $(25 \mathrm{mg} / \mathrm{kg}$ of fluralaner) administered to dogs showed a phlebotomine mortality effect. The phlebotomine mortality ranged 

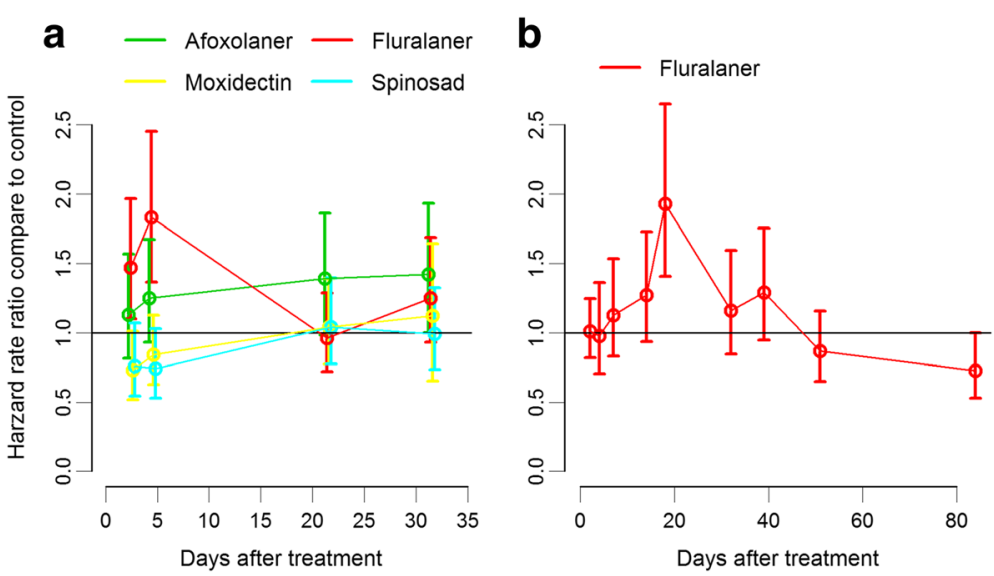

Fig. 2 Hazard rate ratio compared with control for each treatment group and sampling day estimated from a mixed cox proportional hazard model. The variable dog was included as a random effect treatment, days after treatment, and their interaction were covariates. a Trial 1: afoxolaner vs fluralaner vs moxidectin vs spinosad vs control. b Trial 2: fluralaner vs control

Table 3 Trial 2 results from the negative binomial mixed model that included treatment group, sample day and their interaction as fixed effects and dog as random effect. Results are presented as the incidence rate ratio (IRR) which is the increase in the rate of incidence compared with control at each sampling day

\begin{tabular}{llll}
\hline Covariates effect $^{\mathrm{a}}$ & IRR $^{\mathrm{b}}$ & $95 \% \mathrm{Cl}$ & $P$-value \\
\hline Day 2: Control & Reference & - & - \\
Fluralaner & 1.24 & $0.78-1.96$ & 0.35 \\
Day 4: Control & Reference & - & - \\
Fluralaner & 1.40 & $0.90-2.18$ & 0.13 \\
Day 7: Control & Reference & - & - \\
Fluralaner & 1.42 & $0.92-2.19$ & 0.11 \\
Day 14: Control & Reference & - & - \\
Fluralaner & 1.64 & $1.16-2.54$ & $0.025^{*}$ \\
Day 18: Control & Reference & - & - \\
Fluralaner & 1.22 & $0.77-1.91$ & 0.38 \\
Day 32: Control & Reference & - & - \\
Fluralaner & 1.97 & $1.23-3.17$ & $0.004^{*}$ \\
Day 39: Control & Reference & - & - \\
Fluralaner & 0.92 & $0.59-1.44$ & 0.73 \\
Day 51: Control & Reference & - & - \\
Fluralaner & 1.24 & $0.93-1.66$ & 0.14 \\
Day 84: Control & Reference & - & - \\
Fluralaner & 0.93 & $0.68-1.27$ & 0.68 \\
\hline
\end{tabular}

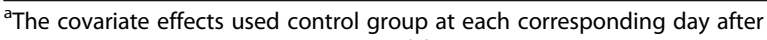
treatment as baseline comparison $[\operatorname{Pr}(>|z|)]$

${ }^{\mathrm{b}}$ Incidence rate ratio, as the ratio between the incidence rate in control group and the incidence rate for each treatment group, at the corresponding sampling day after treatment

"Significance level defined at $\alpha=0.05$
Table 4 Trial 2 results from the Cox proportional hazard model that included treatment group, sample day and their interaction as fixed effects and dog as random effect. Results are presented as the hazard rate ratio (HRR) which is the increase in the hazard rate compared with control at each sampling day

\begin{tabular}{llll}
\hline Covariates effect $^{\mathrm{a}}$ & HRR $^{\mathrm{b}}$ & $95 \% \mathrm{Cl}$ & $P$-value \\
\hline Day 2: Control & Reference & - & - \\
Fluralaner & 0.97 & $0.70-1.36$ & 0.90 \\
Day 4: Control & Reference & - & - \\
Fluralaner & 1.12 & $0.70-1.53$ & 0.43 \\
Day 7: Control & Reference & - & - \\
Fluralaner & 1.27 & $0.94-1.72$ & 0.12 \\
Day 14: Control & Reference & - & - \\
Fluralaner & 1.92 & $1.40-2.64$ & $<0.0001^{*}$ \\
Day 18: Control & Reference & - & - \\
Fluralaner & 1.15 & $0.84-1.59$ & 0.36 \\
Day 32: Control & Reference & - & - \\
Fluralaner & 1.28 & $0.94-1.75$ & 0.10 \\
Day 39: Control & Reference & - & - \\
Fluralaner & 0.86 & $0.65-1.15$ & 0.33 \\
Day 51: Control & Reference & - & - \\
Fluralaner & 0.86 & $0.64-1.15$ & 0.22 \\
Day 84: Control & Reference & - & - \\
Fluralaner & 0.73 & $0.53-1.05$ & 0.09 \\
\hline
\end{tabular}

${ }^{\mathrm{a}}$ The covariate effects used control group at each corresponding day after treatment as baseline comparison $[\operatorname{Pr}(>|z|)]$

${ }^{\mathrm{b}}$ Hazard rate ratio, as the ratio between the hazard rate in control group and the hazard rate for each treatment group, at the corresponding sampling day after treatment

*Significance level defined at $a=0.05$ 
from 60 to $80 \%$ up to 30 days after treatment. This mortality was lower than the mortality reported in fleas. In fleas, the main the target of the product, the mortality reported was 100\% 24 hours after infestation during 12 weeks after treatment $[12,29,30]$. The lower mortality effect found in phlebotomine sand flies comparing with the mortality found in flea studies, besides the differences in the study design [direct feeding (fleas) versus membrane feeding], could be due to their differences in feeding behavior. For example, sand flies ingest $1 \mu \mathrm{l}$ of blood or less [31] while fleas ingest $110 \mu \mathrm{l}$ [32] of blood, sand flies take an average of six minutes to feed on blood [33] while fleas take an average of 25 minutes [34]. These feeding characteristics could influence the amount and the bioavailability of the insecticide.

The other compounds tested: spinosad, afoxolaner, and moxidectin did not show significant increase in phlebotomine mortality. This may be due in part to the low concentration used in this study. For example, we administered spinosad at $45-70 \mathrm{mg} / \mathrm{kg}$; however, previous studies in rodents showed that spinosad treated diet had phlebotomine mortality when administered at 5000 $\mathrm{mg} / \mathrm{kg}$ but no significant mortality was observed at 500 $\mathrm{mg} / \mathrm{kg}$ of spinosad [35]. Nevertheless, spinosad, as well as afoxolaner and fluralaner administered at label concentration showed to have significant lethal effects on triatomines directly feeding on dogs [36]. Fluralaner and afoxolaner killed $100 \%$ of the triatomines for 51 days after oral administration. Lower mortality $(50-70 \%)$ was observed in the spinosad group.

Furalaner showed a significant increase in sand fly mortality in both trials 1 and 2. However, the two trials differ in the onset of action and the duration of it. These differences can be explained by (i) higher phlebotomine mortality in control group in trial 2 (phlebotomine mortality at day $2: 63 \%$, CI 53,72 ) than in trial 1 (day 2: $28 \%$, CI 19,38), and (ii) the influence of food on the pharmacokinetics (PK) of fluralaner. More precisely, for the first trial, the chewable tablet of fluralaner was administered early in the morning before feeding the dogs: the dogs' last meal was about 20 hours before administration. In trial 2, the chewable tablet was administered after the dogs received half of their daily food ration. One PK study showed that when fluralaner is administered with food, its bioavailability is duplicated [20].

The mortality associated to the different treatments observed in this study may be an underestimation. Mortality in the control group 24 hours after blood-feeding was much higher (50\%, Additional file 4: Figure S3) than expected $(20 \%)$ and variable (e.g. phlebotomine mortality in trial 2 range from $34 \%$ in day 32 to $63 \%$ in day 2). The high mortality reduced the power of the trials and the variability may explain the inconsistencies on the effect observed on consecutive sampling days (e.g, statically significant mortality on day 14 and 32 but not on day 21 in trial 2). Despite the high mortality in the control group we demonstrated that systemic insecticides, such as fluralaner have mortality effect against phlebotomine sand flies. Variations of mortality in controls may be due to (i) use membrane feeding instead of direct feeding; (ii) low number of sand flies per membrane feeding; or (iii) phlebotomine environmental temperature or humidity. Reducing mortality in control group may allow better estimation of mortality due to systemic insecticides.

\section{Conclusions}

Despite the limitations, this study shows that a single oral treatment of fluralaner in dogs induces phlebotomine mortality. This would indicate that administering systemic insecticides to dogs could potentially be used to control ZVL, but further work needs to be conducted to define the requirements of compounds to be used in a public health intervention, e.g. insecticide efficacy, duration of the efficacy, route of administration or the percentage of dogs treated in the community. Finally, a clinical trial should be conducted to evaluate the impact of administering systemic insecticides to dogs to control $\mathrm{ZVL}$ in humans in endemic areas.

\section{Additional files}

Additional file 1 Figure S1. Organization chart showing the sampling schedule. (TIFF $108 \mathrm{~kb}$ )

Additional file 2: Table S1. Randomization results. Table S2. Trial 1 sand fly mortality, percentage, and $95 \% \mathrm{Cl}$ at 24, 48, 72, 96 and 120 hours after blood feeding for treatment group and sampling day. Table S3. Trial 2, sand fly mortality, percentage, and its $95 \% \mathrm{Cl}$ at 24, 48, 72, 96 and 120 hours after blood feeding for treatment group and sampling day. Table S4. Trial 1 results from the negative binomial mixed model with treatment group, sample day and their interaction as explanatory variables and dog as random effect. Table S5. Trial 1 Cox proportional hazard mixed model with treatment group, sample day and their interaction as explanatory variables and dog as random effect. Table S6. Trial 2 negative binomial mixed model with treatment group, sample day and their interaction as explanatory variables and dog as random effect. Table S7. Trial 2 Cox proportional hazard mixed model with treatment group, sample day and their interaction as explanatory variables and dog as random effect (DOCX $53 \mathrm{~kb}$ )

Additional file $\mathbf{3}$ Figure S2. Trial 1, sand fly survival after feeding with blood collected from dogs 2, 4, 21 and 31 days after treatment administration. Groups: control (black); afoxolaner (green); fluralaner (red); moxidectin (yellow); and spinosad (blue). (TIFF 66 kb)

Additional file 4 Figure S3. Sand fly mortality percentage and its 95\% $\mathrm{Cl}$ at 24 hours after blood feeding for each treatment group and sampling day. A: Trial 1: afoxolaner vs fluralaner vs moxidectin vs spinosad vs control. B: Trial 2: fluralaner vs control (TIFF $65 \mathrm{~kb}$ )

Additional file 5 Figure S4. Trial 2, sand fly survival after feeding with blood collected from dogs $0,2,4,7$ 14, 18, 32, 39, 51 and 84 days after treatment administration by treatment groups: control (black), fluralaner (red). (TIFF $37 \mathrm{~kb}$ )

\section{Abbreviations}

GABA: Gamma-aminobutyric acid; HRR: Hazard rate ratios; IRR: Incidence rate ratio; PK: Pharmacokinetics; ZVL: Zoonotic visceral leishmaniasis 


\section{Acknowledgements}

We thank Rafael Escar, the owner of the breeding kennel, who kindly collaborated in this project. ISGlobal is a member of the CERCA Programme, Generalitat de Catalunya.

\section{Funding}

This research is funded by the European Union's H2020 Programme under the MSCA GA no. 642609

\section{Availability of data and materials}

The datasets used and/or analyzed during the current study are available from the corresponding author upon reasonable request.

\section{Authors' contributions}

Design: SAG, AP, JACH and JLC. Experiments: SAG, PPP, AEG, RVOV and POH. Analysis: SAG and AP. Manuscript: SAG and AP. All authors read and approved the final manuscript.

\section{Ethics approval}

Approvals for the animal experiments were obtained from the Universidad de Zaragoza (UNIZAR), Zaragoza, España (Comisión Ética Asesora para la Experimentación Animal, ref. PI05/16) and the University of Barcelona, España, Comite Etic d'Experimentación Animal (CEEA no. 565/15). All anima handling and bioassay procedures, and the maintenance of the $P$. papatasi colony, followed protocols approved by the ethical committee of the University of Zaragoza, Spain.

\section{Consent for publication}

$$
\text { Not applicable. }
$$

\section{Competing interests}

The authors declare that they have no competing interests.

\section{Publisher's Note}

Springer Nature remains neutral with regard to jurisdictional claims in published maps and institutional affiliations.

\section{Author details}

'ISGlobal, Barcelona Ctr. Int. Health Res. (CRESIB), Hospital Clínic-Universitat de Barcelona, Barcelona, Spain. '2Department of Animal Pathology, Faculty of Veterinary Medicine at the University of Zaragoza, Zaragoza, Spain.

\section{Received: 5 December 2017 Accepted: 26 March 2018}

\section{Published online: 05 April 2018}

\section{References}

1. Alvar J, Vélez ID, Bern C, Herrero M, Desjeux P, Cano J, et al. Leishmaniasis worldwide and global estimates of its incidence. PLoS One. 2012;7:e35671.

2. Aronson N, Herwaldt BL, Libman M, Pearson R, Lopez-Velez R, Weina P, et al. Diagnosis and treatment of leishmaniasis: clinical practice guidelines by the Infectious Diseases Society of America (IDSA) and the American Society of Tropical Medicine and Hygiene (ASTMH). Clin Infect Dis. 2017;96(1):24-45.

3. Quinnell RJ, Courtenay O. Transmission, reservoir hosts and control of zoonotic visceral leishmaniasis. Parasitology. 2009;136:1915-34.

4. Killick-Kendrick R. Phlebotomine vectors of the leishmaniases: a review. Med Vet Entomol. 1990:4:1-24.

5. Courtenay O, Quinnell RJ, Garcez LM, Shaw JJ, Dye C. Infectiousness in a cohort of Brazilian dogs: why culling fails to control visceral leishmaniasis in areas of high transmission. J Infect Dis. 2002;186:1314-20.

6. Gomez SA, Picado A. Systemic insecticides used in dogs: potential candidates for phlebotomine vector control? Trop Med Int Health. 2017;22:755-64.

7. Dryden MW, Canfield MS, Kalosy K, Smith A, Crevoiserat L, MCGrady JC, et al. Evaluation of fluralaner and afoxolaner treatments to control flea populations, reduce pruritus and minimize dermatologic lesions in naturally infested dogs in private residences in west central Florida USA. Parasit Vectors. 2016;9:365.

8. Crosaz O, Chapelle E, Cochet-Faivre N, Ka D, Hubinois C, Guillot J. Open field study on the efficacy of oral fluralaner for long-term control of flea allergy dermatitis in client-owned dogs in lle-de-France region. Parasit Vectors. 2016;9:174.
9. Blagburn BL, Young DR, Moran C, Meyer JA, Leigh-Heffron A, Paarlberg T, et al. Effects of orally administered spinosad (Comfortis $\left.{ }^{\circledR}\right)$ in dogs on adult and immature stages of the cat flea (Ctenocephalides felis). Vet Parasitol. 2010;168:312-7.

10. Blagburn BL, Dillon AR, Arther RG, Butler JM, Newton JC. Comparative efficacy of four commercially available heartworm preventive products against the MP3 laboratory strain of Dirofilaria immitis. Vet Parasitol. 2011; 176:189-94.

11. Pfister K, Armstrong R. Systemically and cutaneously distributed ectoparasiticides: a review of the efficacy against ticks and fleas on dogs. Parasit Vectors. 2016;9:436.

12. Taenzler J, Wengenmayer C, Williams H, Fourie J, Zschiesche E, Roepke RK, et al. Onset of activity of fluralaner (BRAVECTO ${ }^{\mathrm{TM}}$ ) against Ctenocephalides felis on dogs. Parasit Vectors. 2014;7:567.

13. Beugnet F, deVos C, Liebenberg J, Halos L, Fourie J. Afoxolaner against fleas: immediate efficacy and resultant mortality after short exposure on dogs. Parasite. 2014:21:42.

14. Becskei C, De Bock F, Illambas J, Cherni JA, Fourie JJ, Lane M, et al. Efficacy and safety of a novel oral isoxazoline, sarolaner (Simparica ${ }^{\mathrm{TM}}$ ), for the treatment of sarcoptic mange in dogs. Vet Parasitol. 2016;222:56-61.

15. Bowman DD. Heartworms, macrocyclic lactones, and the specter of resistance to prevention in the United States. Parasit Vectors. 2012;5:138,

16. Mascari TM, Stout RW, Foil LD. Evaluation of three feed-through insecticides using two rodent and two sand fly species as models. J Am Mosq Control Assoc. 2012;28:260-2.

17. Ingenloff K, Garlapati R, Poché D, Singh MI, Remmers JL, Poché RM. Feedthrough insecticides for the control of the sand fly Phlebotomus argentipes. Med Vet Entomol. 2013;27:10-8.

18. Derbali M, Polyakova L, Boujaâma A, Burruss D, Cherni S, Barhoumi W, et al. Laboratory and field evaluation of rodent bait treated with fipronil for feed through and systemic control of Phlebotomus papatasi. Acta Trop. 2014;135:27-32.

19. Walther FM, Allan MJ, Roepke RK, Nuernberger MC. The effect of food on the pharmacokinetics of oral fluralaner in dogs. Parasit Vectors. 2014;7:84.

20. Suresh K, Chandrashekara S. Sample size estimation and power analysis for clinical research studies. J Hum Reprod Sci. 2012;5:7-13.

21. Altman DG, Schulz KF. Concealing treatment allocation in randomised trials. BMJ. 2001;323:446-7.

22. Matts JP, Lachin JM. Properties of permuted-block randomization in clinical trials. Control Clin Trials. 1988:9:327-44.

23. R Core Team. R: A language and environment for statistical computing. Vienna: R Foundation for Statistical Computing; 2015.

24. Cosgrove JB, Wood RJ, Petrić D, Evans DT, Abbott RH. A convenient mosquito membrane feeding system. J Am Mosq Control Assoc. 1994;10:434-6.

25. Alexander N. Analysis of parasite and other skewed counts. Trop Med Int Health. 2012;17:684-93.

26. Venables WN, Ripley BD. Modern Applied Statistics with S. Fourth ed. New York: Springer; 2002

27. Therneau TM, Grambsch PM. Modeling Survival Data: Extending the Cox Model. New York: Springer; 2000

28. Therneau TM. coxme: Mixed effects Cox models. 2015. https://cran.r-project. org/web/packages/coxme/index.html. Accessed 27/06/2107.

29. Rohdich N, Roepke RK, Zschiesche E. A randomized, blinded, controlled and multi-centered field study comparing the efficacy and safety of Bravecto ${ }^{\mathrm{TM}}$ (fluralaner) against Frontline ${ }^{\mathrm{TM}}$ (fipronil) in flea- and tick-infested dogs. Parasit Vectors. 2014:7:83

30. Dryden MW, Smith V, Bennett T, Math L, Kallman J, Heaney K, et al. Efficacy of fluralaner flavored chews (Bravecto ${ }^{\oplus}$ ) administered to dogs against the adult cat flea, Ctenocephalides felis felis and egg production. Parasit Vectors. 2015;8:364.

31. Daba S, Daba A, Shehata MG, El Sawaf BM. A simple micro-assay method for estimating blood meal size of the sand fly, Phlebotomus langeroni (Diptera: Psychodidae). J Egypt Soc Parasitol. 2004:34:173-82.

32. Dryden MW, Gaafar SM. Blood consumption by the cat flea, Ctenocephalides felis (Siphonaptera: Pulicidae). J Med Entomol. 1991;28:394-400.

33. Cadiergues MC, Hourcq P, Cantaloube B, Franc M. First bloodmeal of Ctenocephalides felis felis (Siphonaptera: Pulicidae) on cats: time to initiation and duration of feeding. J Med Entomol. 2000;37:634-6.

34. Rogers ME, Bates PA. Leishmania manipulation of sand fly feeding behavior results in enhanced transmission. PLoS Pathog. 2007;3:e91. 
35. Mascari TM, Stout RW, Foil LD. Laboratory evaluation of oral treatment of rodents with systemic insecticides for control of bloodfeeding sand flies (Diptera: Psychodidae). Vector-Borne Zoonotic Dis. 2012;12:699-704.

36. Loza A, Talaga A, Herbas G, Canaviri RJ, Cahuasiri T, Luck L, et al. Systemic insecticide treatment of the canine reservoir of Trypanosoma cruzi induces high levels of lethality in Triatoma infestans, a principal vector of Chagas disease. Parasit Vectors. 2017;10:344.

Submit your next manuscript to BioMed Central and we will help you at every step:

- We accept pre-submission inquiries

- Our selector tool helps you to find the most relevant journal

- We provide round the clock customer support

- Convenient online submission

- Thorough peer review

- Inclusion in PubMed and all major indexing services

- Maximum visibility for your research

Submit your manuscript at www.biomedcentral.com/submit 